\title{
Firmware Enhancements for BYOD-Aware Network Security
}

\author{
M. Pedone ${ }^{1 *}$, K. Kanev ${ }^{2}$, P. Bottoni ${ }^{1}$, D. Vitali ${ }^{1}$, A. Mei $^{1}$ \\ ${ }^{1}$ Sapienza University of Rome, Piazzale Aldo Moro 5, 00185 Roma, Italy \\ ${ }^{2}$ Shizuoka University,3-5-1 Johoku, Naka-ku, Hamamatsu, 432-8011, Japan \\ *massimiliano.pedone@uniromal.it
}

\begin{abstract}
In today's connected world, users migrate within a complex set of networks, including, but not limited to, $3 \mathrm{G}$ and $4 \mathrm{G}$ (LTE) services provided by mobile operators, Wi-Fi hotspots in private and public places, as well as wireless and/or wired LAN access in business and home environments. Following the widely expanding Bring Your Own Device (BYOD) approach, many public and educational institutions have begun to encourage customers and students to use their own devices at all times. While this may be cost-effective in terms of decreased investments in hardware and consequently lower maintenance fees on a long-term basis, it may also involve some security risks. In particular, many users are often connected to more than one network and/or communication service provider at the same time, for example to a $3 \mathrm{G} / 4 \mathrm{G}$ mobile network and to a Wi-Fi. In a BYOD setting, an infected device or a rogue one can turn into an unwanted gateway, causing a security breach by leaking information across networks. Aiming at investigating in greater detail the implications of BYOD on network security in private and business settings we are building a framework for experiments with mobile routers both in home and business networks. This is a continuation of our earlier work on communications and services with enhanced security for network appliances.
\end{abstract}

Keywords: Network Security, Modeling, Diagnostics, Learning Techniques.

\section{Introduction}

In our current study, we shift the focus to BYOD and mobile Wi-Fi routers, which require more advanced functionality and interfacing. In particular, we explore extensions of existing community firmware necessary for BYOD-aware, secure, seamless integration of the mobile communication appliances in private, public, and business networks, as carried on by their owners. The multiplatform firmware will also allow a carefully designed single GUI to be made available to users of communication appliances from different producers, irrespectively of their make and model.

While this is still work in progress, we are considering the possible applicability of our approach to the LM-28225 Master's Programme in Computer Science at Sapienza [1]. The specialized BYOD-based framework, for example, could be instrumental in a Networks and Security course [2], while the advanced BYOD-aware communication 
model would be more relevant to a Software Engineering course [3].

The paper follows with Section 2 which provides an overview of typical security problems in a university network used by thousands of people every day. Section 3 considers opportunities for securing personal devices when their use is intended in third party networks, while Section 4 concludes the paper and discusses future work.

\section{Large Networks and Security}

Network access has never been considered entirely secure. As a large number of devices share the same network, one has to be vigilant against many security threats. In fact, any neighboring device can get infected by malicious software and perpetrate all kinds of attack unbeknownst to its owner.

As all devices share the same radiofrequency, all data, sent and received, is potentially accessible by others. Indeed, as wireless transmissions are inherently broadcast, any device can monitor and record them. Although access to the information embedded in such communications is protected by encryption and various user authentication mechanisms, its security cannot be guaranteed [4]. Security threats are much higher though, in public network environments and open access community networks.

Every day, students, faculty members, and other personnel use university networks both to exchange sensitive information and to access cloud resources and external websites [5]. In this work we consider the Sapienza University campus wireless network as a reference and test bed environment. Our university domain consists of over 3,000 wired PCs, workstations, and laboratory computers and services over 10,000 of Wi-Fi based mobile connections daily. In Figures 1 and 2 we show summaries of the detected security threats and virus infection incidents in a recent 7-day period (dates withhold on security grounds).

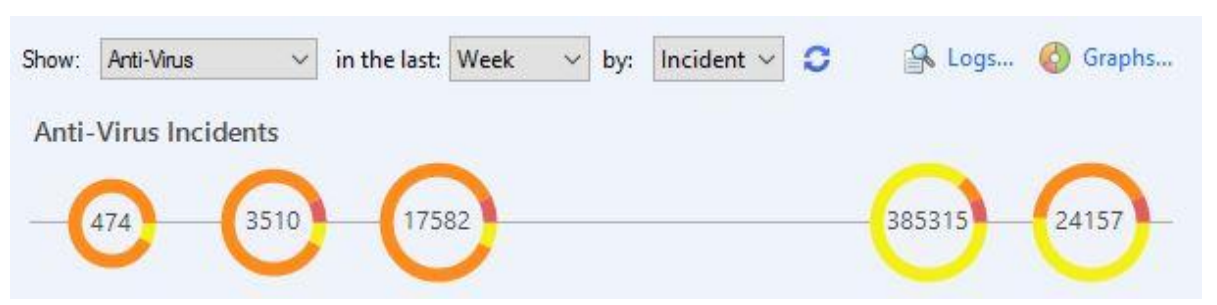

Fig. 1. Number of security incidents over a 7-day period. Risk levels ranging from yellow to red indicate Low, Medium, and Critical values as defined in the Common Vulnerability Scoring System (CVSS) database.

Any virus protection software, however, impacts the performance of the firewall core on which it is installed [6]. The potential slowdown depends on the complexity of the protection and the nature of the traffic blend. HTTP server access, for example, generates a lot of traffic in contrast to the FTP and Telnet traffic which is more limited while all those protocols require different levels of inspection.

The relationships between the threat severity levels and their relevant protective measures with respect to performance are outlined in Table 1. 


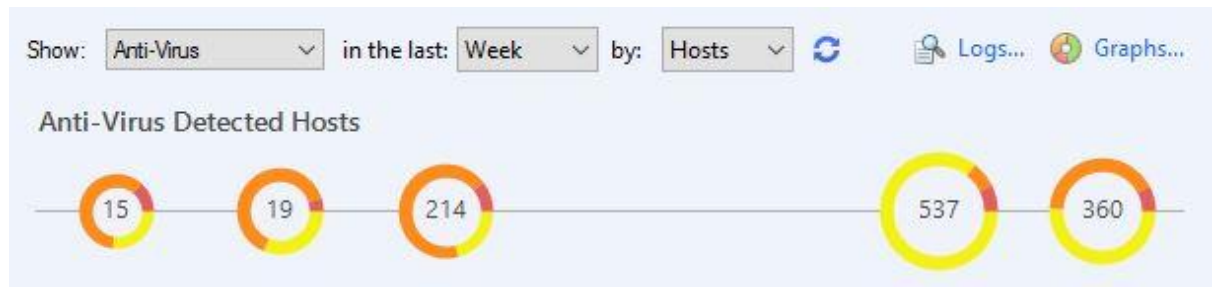

Fig. 2 Number of infected hosts over a 7-day period. Virus infections are classified as adware (low severity) and malware (medium or high severity) as per the severity levels of antivirus and antibot protections given in Table 1 .

Table 1. Severity levels of antivirus and antibot protections [7]

\begin{tabular}{ll}
\hline Severity Levels & Case \\
\hline Very Low & $\begin{array}{l}\text { All protections which do not cause any performance degradation. } \\
\text { - New protections are not added to this category without perfor- } \\
\text { mance tests. }\end{array}$ \\
\hline Low & $\begin{array}{l}\text { All simple signatures over any protocol, which have very unique } \\
\text { traffic patterns. }\end{array}$ \\
\hline Medium & All HTTP Client protections, which use complex detection logic. \\
& - All protocol parsers, which perform protocol anomaly over PSL. \\
& All signatures executed on HTTP responses. \\
\hline High & Protections which are executed on all ports. \\
& Performs extremely heavy and complex detection logic. For exam- \\
& ple, decoding of RC4 encryption.
\end{tabular}

\subsection{Network security threats}

From a technical point of view, users sharing the same network segment are also sharing one and the same broadcast domain. While various security policies may be implemented by network operators within the domain, establishing and managing a security perimeter is usually conducted at the inter-domain communication level, where firewalls and other specialized network access control equipment is employed.

The security mechanisms that are generally available in advanced network switches, routers, and other communication equipment are usually not applicable or are simply missing within the broadcast domain itself. Such intra-domain communications are indeed rarely moderated and, in most cases, the responsibility is left to individual users to install and properly maintain the necessary security tools. Unfortunately, given the complexity of the current network threats, only a small portion of the connected users are in a position to properly install and maintain such tools on the large diversity of communication devices they use [8]. To address this situation we propose the use of inexpensive, privately owned Mobile Network Appliances (MNAs) that establish a security perimeter for each individual network user in a unified way. 


\subsection{Security and communication protocols}

In business environments, various attempts to improve the security of private devices have been made through the selection of more appropriate network protocols, advanced packet routing and firewalling, and other responsive network solutions [6]. Unfortunately, for a more complete coverage of existing users and their devices, many network security features have to be excluded or kept off, in particular when legacy devices are used. For example, the IEEE 802.1x standard provides a secure, per port authentication protocol, but legacy hardware and even recent devices based on certain chipsets do not support it, so network operators often opt to implement other less secure network access mechanisms.

In many cases, users are admitted to the Wi-Fi network without authentication, e.g. via captive portals. With this technology users are authenticated at a later stage trough a dedicated Web page, just before granting them access to the Internet. This implies insecure communication between the mobile device and the access point at the initial stages of the communication, which may allow eavesdropping and collection of login information by bystanders. Employing Wi-Fi Protected Access (WPA) or another secure Wi-Fi access protocol since the very beginning of the session would certainly be a much better solution which, unfortunately, may be difficult to enforce [4].

In our security model, we address this problem by employing private MNAs serving as security gateways and providing encrypted access to the AP for all user devices despite the existing inherent limitations. In our model, the secure channel between the MNA and the AP is established by the MNA itself based on its own credentials.

\subsection{Man in the middle attacks}

The Man-in-the-Middle (MITM) attack is a complex technique allowing a malicious user to eavesdrop on communications between other customers. Such an attack may lead to severe consequences in terms of privacy and confidentiality when user information is stolen and used for malicious purposes. MITM attacks are widespread in large networks and have in fact been often detected in the Sapienza network. While mounting a MITM attack, the malicious agent impersonates a trustworthy authority or a third party that may be a gateway or an authentication host. Since this remains undetected by the attacked device, its user will believe to have been prompted for authentication credentials by the legitimate authority. In reality, however, every communication the attacked device sends or receives will be intercepted and altered by the malicious agent employing it for breaking the security settings of the user.

Secure protocols like Secure Socket Layer and Transport Layer Security are often ineffective with respect to MITM attacks. Indeed, advanced MITM techniques allow attackers to divert encrypted traffic (e.g. https) to clear text traffic (e.g. http). While such attacks can only be mounted on Web servers that support both clear text and encrypted traffic, this remains one of the most common configurations on the net. 


\subsection{Malware threats}

While a network scan could be benign in its nature, it is often recognized as the first step in a chain of attacks on a network. Users can employ publicly available net scan application and host maintenance script for both discovering the hosts connected to the network and identifying the services exposed by those hosts [9]. Such software allows malicious users to discover the programs installed on the scanned hosts and to proceed with identifying the specific versions that may be vulnerable. Through this technique, malicious users can find exploits allowing them unauthorized privileged access to just one host on the attacked network which may jeopardize the safety of all computers in the security perimeter domain.

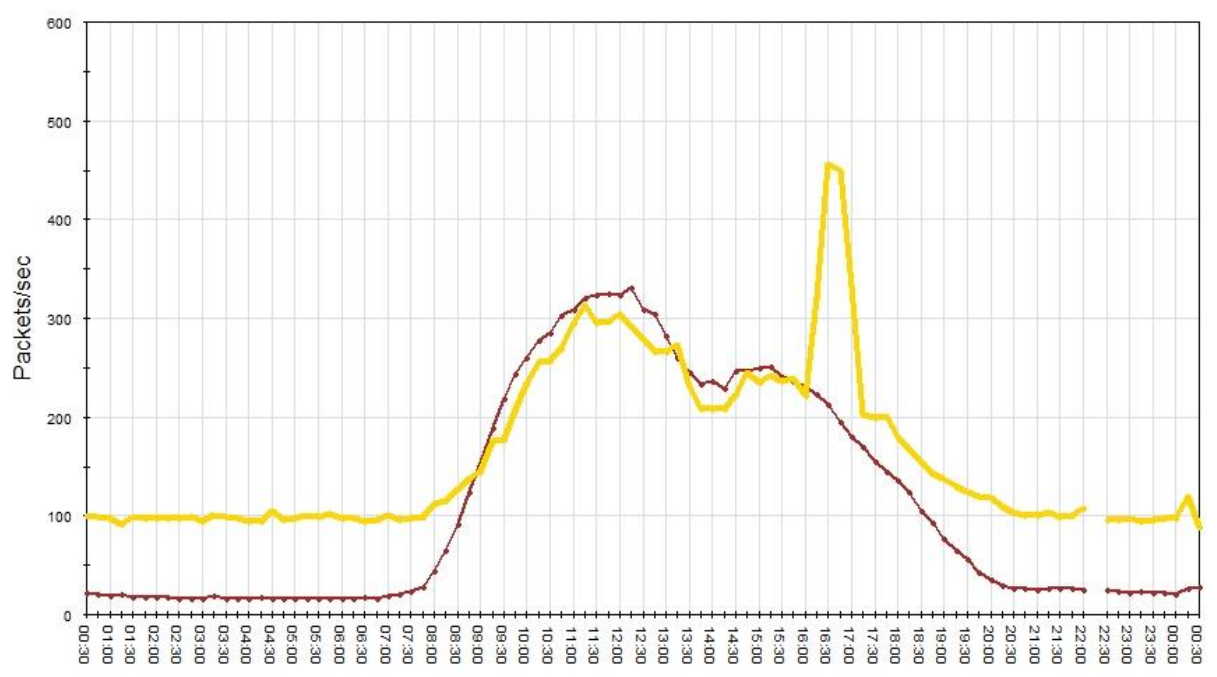

Fig. 3. The activity of the "wanna cry" virus: the yellow curve represents the dropped packets while the red one indicates the number of related connections during the attack. The peak of the dropping indicates the adequate reaction of the updated and properly patched firewall system.

The recent incidents of virus attacks and consequent security breaches affecting entire domains clearly indicate that users are confronting increasing dangers in their daily networking. In business oriented network environments this problem is usually addressed through timely virus protection patches and more advanced packet filtering. For better understanding of the threat identification and consequent data exclusion at the time of a network attack, we have provided in Figure 3 the graph of all virus related connections and respective dropped packets over a 24 hour period.

The "wanna cry" virus (WCv) is a malware which infects a storage device on an unpatched system and asks for a ransom to decrypt the contents of the stored files. It exploits the EternalBlue vulnerability to install a backdoor called DoublePulsar $(\mathrm{DPb})$, both supposedly developed by the U.S. National Security Agency. The WCV first attempts to load the infection through the public key infrastructure (.pki). If . pki is not found, WCV creates a key pair by "CriptGenKey", saves the public part of the key, and uses the master key to encrypt the private part stored in a file 
with .eki extension. Then "CryptGenRandom" is used to generate separate Advanced Encryption Standard (AES) keys for every file that is encrypted. Each AES key is encrypted using the previously generated public key and is merged into its corresponding file. To decrypt the resulting files, the unlucky owner needs the private part of the master key, for which a ransom is requested. In the course of the above and afterwards, the $\mathrm{DPb}$ keeps sending out scans and mounts attacks to propagate the worm. The yellow peak in Figure 3 indicates that the Sapienza firewall has successfully detected and dropped the large number of connections initiated by the DPb.

\section{$3 \quad$ Securing Your Own Device}

In the context of all the network threats discussed so far, securing your own device appears to be far from trivial task. With respect to this, however, a dedicated MNA, hosting an embedded system configured with network security in mind, can incorporate a number of essential network control and security applications and tools. This will ensure a centralized deep packet inspection and monitoring of related network activities as a basis for timely feedback and support of the MNA owner by alerts and flagging of suspicious traffic [1].

While such network control and security applications can be directly installed on the user devices, in such a case they will consume computing resources on each device and will need separate maintenance and updates on a per device basis. Furthermore, in case of infection, the malicious software can alter the security settings of the applications directly installed on the device, creating serious security breaches. As MNA-based security applications and tools are installed in the read-only flash memory of the embedded device, they cannot be altered by any malicious software.

Moreover, the MNA approach allows users to establish and maintain secure VPN channels between the MNA device and the owner's home or business network. Thus, user communications are secure at all times, regardless possible differences in the setups and corresponding security levels supported by the employed devices [10].

The MNA approach extends our earlier work with augmented embedded systems for communication appliances. In this approach, as sketched in Figure 4, we are considering mobile routers, with community DD-WRT firmware as MNAs, which are not confined to a home or laboratory environment. As such MNAs are constantly with the user, they can help enforcing a consistent security policy across several network environments, thus ensuring better protection of users in the context of the BYOD model.

First of all, DD-WRT features a fully fledged State Packet Inspection (SPI) firewall with additional filters for cookies, Java applets, and ActiveX content. It can keep track of all incoming and outgoing traffic locally up to a certain extent along with a full record on a remote log server. A special single click options for impeding Wide Area Network (WAN) Denial of Service (DoS) and brute force attacks is also included. Even if these firewall features are turned off, the DD-WRT will isolate the user from the WAN by acting as a Network Address Translations gateway [11]. In such a scenario, the MNA equipment is directly exposed to the network, while the mobile devices of the user are masqueraded. The DD-WRT router mediates each outgoing 
connection and inhibits unrelated and undesired ingoing connections. Implicitly, the mobile devices of the user connected to the Internet through the MNA remain protected and no direct connections to them from the outside world can be established.

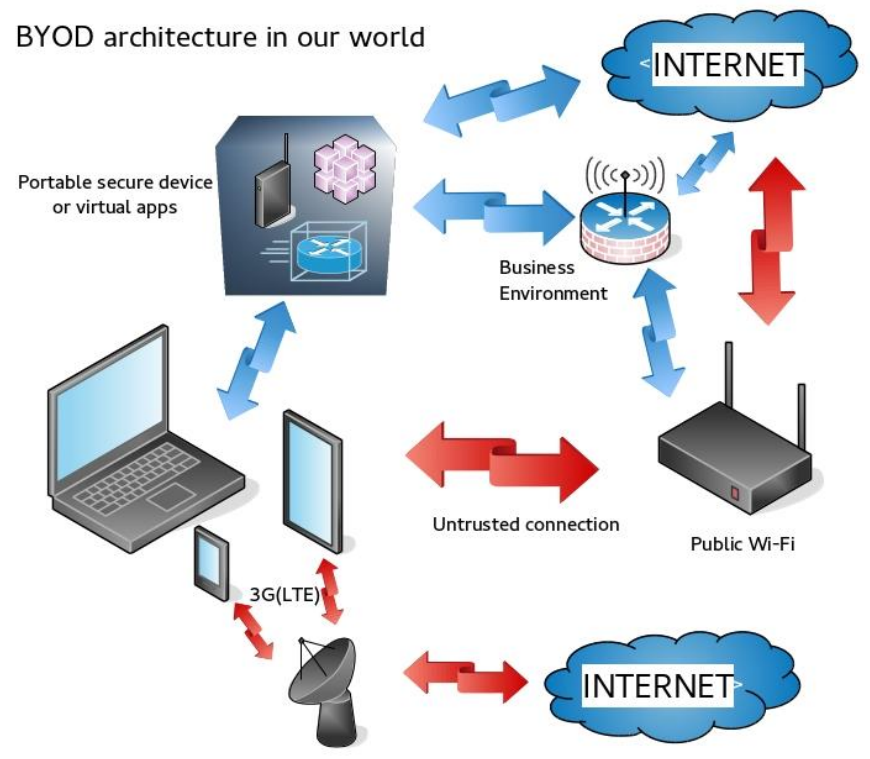

Fig. 4. MNA access for secure BYOD communications. The red arrows represent unsecure communications while the blue arrows correspond to connections secured through MNA. The Wi-Fi component of the DD-WRT software supports multiple physical and virtual wireless interfaces. This makes it possible to create a number of transparently connected and/or fully isolated network segments as dictated by the security requirements of any specific application. As discussed earlier, suggested way to avoid clear text credential exchanges is to employ WPA or WPA2 with AES cryptography. While the WPA/WPA2-Personal version is more appropriate for individual use, the WPA/WPA2-Enterprise version is also supported through a RADIUS server. In addition to this, MAC based control employing black/white lists is also available.

With respect to VPN connectivity, DD-WRT can act as both Point-to-Point Tunneling Protocol (PPTP) and/or OpenVPN server and client simultaneously [10]. The OpenVPN communications are considered more secure as they are enhanced by verification of server and client certificates. Last, but not least, mobile routers with SD memory slots can be turned into Network Attached Storage (NAS) servers accessible by all mobile devices of the MNA owner.

\section{Conclusions and future work}

We have considered BYOD and mobile Wi-Fi routers, which require advanced functionality and interfacing. In particular we are exploring the extensions of the existing 
community firmware that are necessary for BYOD-aware secure seamless integration of the mobile communication appliances in private, public, and business networks as they are carried by their owners. A multiplatform firmware will also allow a carefully designed single GUI to be made available to users of communication appliances from different producers, irrespectively of their make and model.

While this is still work in progress, we are considering the possible applicability of our approach to the LM-28225 Master's Program in Computer Science at Sapienza. The specialized BYOD-based framework, for example, could be instrumental in a Networks and Security course, while the advanced BYOD-aware communication model would be more relevant to a Software Engineering course.

\section{Acknowledgements}

Part of the work reported in this article is supported by the 2017 Cooperative Research Project at Research Center of Biomedical Engineering and Research Institute of Electronics, Shizuoka University.

\section{References}

1. Kanev, K., Mei, A., Bottoni, P., Home Communications and Services with Enhanced Security: Augmented Embedded Systems for Communication Appliances as an Educational Platform, JAP. Journal of Applied Physics Conf. Proc., Vol. 4, 2016, pp.011615(1-5).

2. Heldenbrand, D., Carey, C., The Linux Router - an Inexpensive Alternative to Commercial Routers in the Lab, J. of Comp. Sc. in Colleges, Vol.23, No.1, October, 2007, pp.127-133.

3. Jasani, H., Vendor Neutral Hands-on Labs using Open-Source Products for Wireless Networks Courses, Proc. FIE 2010, pp.S3F1-6.

4. Antoniewicz, B., 802.11 Attacks, Available Online: http://download.aircrack-ng.org/wikifiles/doc/wireless_basics_and_tutorials/802.11\%20Attacks.pdf

5. Villa, A., Varki, E., Characterization of a campus internet workload, Proc. CATA 2012, pp. 140-148.

6. Athanasiades, N., Abler, R., Levine, J., Owen, H., Riley, G., Intrusion Detection Testing and Benchmarking Methodologies, Proc. IWIA'03, Darmstadt, Germany, March 24, 2003.

7. Check Point Software Technologies, Officiial Web Site, IPS, Anti-Bot, and Anti-Virus Protections Severity, Performance, and Confidence Levels, Available Online: https://supportcenter.checkpoint.com/supportcenter/portal?eventSubmit_doGoviewsolutio ndetails $=\&$ solutionid $=$ sk 116254

8. Meurisch C., Seeliger A., Schmidt B., Schweizer I., Kaup F., Mühlhäuser M. (2015) Upgrading Wireless Home Routers for Enabling Large-Scale Deployment of Cloudlets, In: Mobile Computing, Applications, and Services, LNICST, vol. 162, Springer, pp.12-32.

9. Heffner, C., Remote Attacks Against SOHO Routers, Available Online: https://defcon.org/images/defcon-18/dc-18-presentations/Heffner/DEFCON-18-HeffnerRouters-WP.pdf

10. Hall, M., Performance Analysis of OpenVPN on a Consumer Grade Router, Available Online: http://www.cse.wustl.edu/ jain/cse567-08/ftp/ovpn/index.html

11. Mortier, R., Rodden, T., Tolmine, P., Lodge, T., Spencer, R., Crabtree, Homework: Putting Interaction into the Infrastructure, Proc. UIST'12, Cambridge, pp.197-206. 